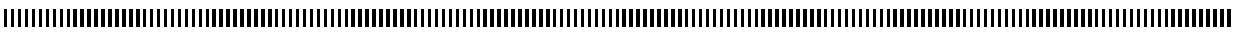

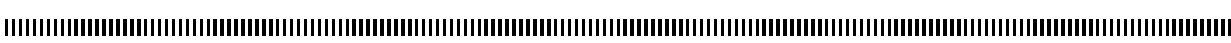

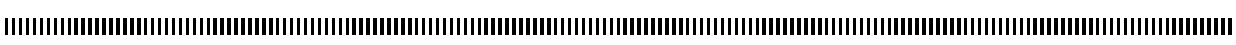

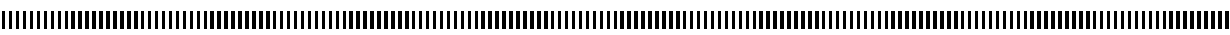

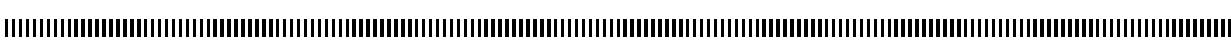

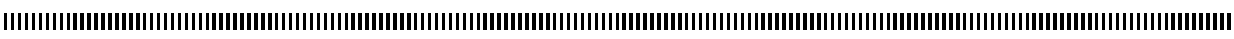

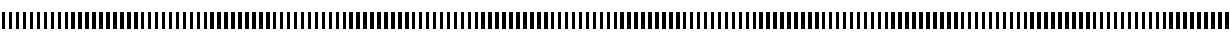

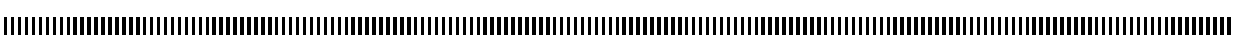

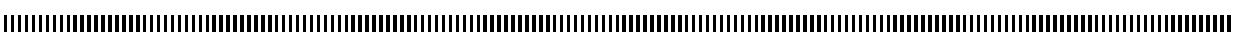

\title{
Optimal control of a parabolic solar collector
}

\author{
Nihale El Boukhari - El Hassan Zerrik \\ MACS Team \\ Faculty of Sciences - Moulay Ismail University \\ B.P. 11201 Zitoune, Meknes \\ Morocco \\ elboukhari.nihale@gmail.com, zerrik3@yahoo.fr
}

|

ABSTRACT. The aim of this paper is to study an optimal control problem for a parabolic solar collector. We consider a bilinear distributed model, where the control models the velocity of the heat-transfer fluid. We prove the existence of an optimal control, and we derive a necessary optimality condition. Then we give an algorithm for the computation of the optimal control. The obtained results are illustrated by simulations of the collector model, using data of Ain Beni Mathar solar plant in Morocco.

RÉSUMÉ. L'objet de cet article est d'étudier un problème de contrôle optimal d'un collecteur solaire parabolique. On considère un modèle bilinéaire distribué, où le contrôle modélise la vitesse du fluide caloporteur. On démontre l'existence d'un contrôle optimal, et on établit une condition nécessaire d'optimalité. Ensuite, on donne un algorithme pour l'implémentation numérique du contrôle optimal. Les résultats obtenus sont illustrés à travers des simulations numériques, en utilisant les données de la station solaire Ain Beni Mathar au Maroc.

KEYWORDS : Bilinear systems, Semigroups, Optimal control, Solar collector, Parabolic trough reflector.

MOTS-CLÉS : Systèmes bilinéaires, Semi-groupes, Contrôle optimal, Collecteur solaire, Réflecteur parabolique. 


\section{Introduction}

Renewable energy is a clean and sustainable alternative to fossil energy. It is a promising technology to face the increasingly global demand for energy and the fluctuating prices of fossil fuels. Particularly, solar thermal energy (STE) is an abundant energy resource, which consists in collecting sunlight to generate thermal or electric energy. The efficiency of solar energy is affected by the position of the sun and by the weather. In this context, the geographic position of Morocco enables the exploitation of solar thermal energy with highly efficient power stations, which significantly reduces electricity production costs. Land availability is also an important factor that gives Morocco a tremendous potential to successfully invest in solar energy.

In this work, we are interested in the optimization of a parabolic power plant performance. Power plants usually use parabolic mirrors to concentrate sunlight onto a receiver tube, containing a heat-transfer fluid, which is often a synthetic oil. The mirrors (or reflectors) change their position along the day from east to west to collect a maximum of sunlight. The receiver tube is enclosed in a vacuum glass envelope. The vacuum significantly reduces the heat losses. Once the fluid is heated by solar irradiation, it is carried to a heat engine, where the heat energy is converted to electricity via steam turbines. For a maximal efficiency of the heat engine, the fluid temperature has to be close to a required level $T_{d} \simeq 673.15 \mathrm{~K}\left(\simeq 400^{\circ} \mathrm{C}\right)$. The fluid temperature depends on the intensity of solar irradiation, on the optical properties of the reflectors, and on the fluid velocity. To drive the fluid temperature close to the required level, it is imperative to determine the optimal fluid velocity, which can be achieved via minimizing a given functional.

This is the aim of this paper. We consider an optimal control problem, where the control stands for the velocity of the heat-transfer fluid. The optimal control to be searched is the minimizer of a quadratic cost functional. The heat balance within the collector is modeled by a distributed bilinear system, written as $\dot{y}(t)=A y(t)+u(t) B y(t)+b(t)$. A rich literature is devoted to the optimal control of such systems (see for instance $[1,4,7,11,13,14]$ ). However, in previous works, the control operator $B$ is usually assumed to be relatively bounded with respect to the dynamics operator $A$. By contrast, the model studied here is a bilinear system whose operator $A$ is bounded, while the control operator $B$ is unbounded, and is the generator of a strongly continuous semigroup. Thereby, the optimal control problem will be studied using a new method, based on the tools of semigroup theory.

The paper is organized as follows. Section 2 is devoted to the description of the distributed bilinear system, modeling the evolution of the fluid temperature. In section 3, we prove the existence of optimal controls, and we derive a necessary optimality condition. In section 4, we provide simulations of the solar collector model, using data of Ain Beni Mathar solar plant in Morocco.

\section{The bilinear model}

We consider a parabolic solar collector, as depicted in Fig. 1. The heat-transfer fluid flows through a metallic receiver tube of length $L$, which is enclosed in a concentric glass envelope. Both tubes are located in the focal line of a parabolic mirror, which concentrates sunlight towards the tubes.

The following assumptions are made on the basis of empirical estimations : 


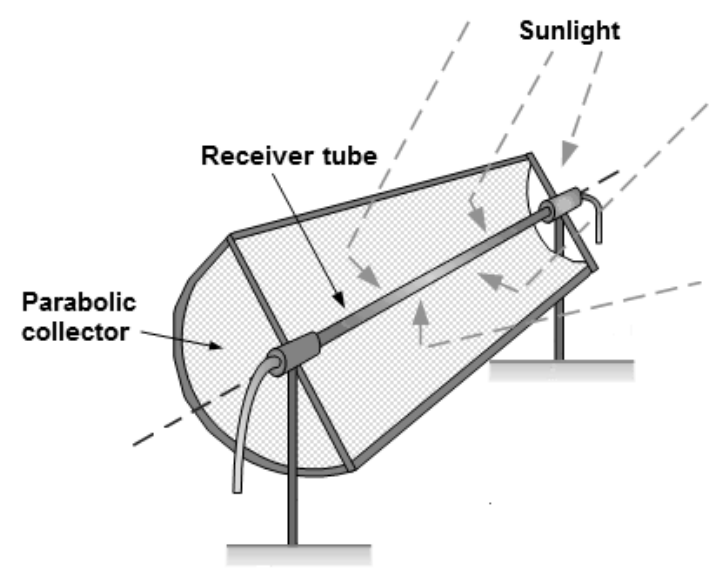

Figure 1. Cylindro-parabolic solar collector [2]

1) The fluid is incompressible, and the fluid flow is laminar, then the fluid velocity is space-independent, and depends only on time.

2) The fluid velocity and temperature are uniformly distributed over the tube section, hence the receiver tube may be modeled by a one-dimensional domain $] 0, L[$.

3) There is perfect vacuum separating the receiver tube and the glass envelope, then the heat loss caused by the glass tube is negligible.

Upon the above assumptions, it has been proved, in subsection 3.3.b of [6], that the heat balance within the solar collector may be modeled by a system of two partial differential equations on $] 0, L\left[\right.$, describing the temperature of the fluid $T_{f}(\mathrm{~K})$ and that of the receiver tube $T_{m}(\mathrm{~K})$, and given by :

$$
\begin{cases}\frac{\partial T_{f}}{\partial t}(x, t)=a T_{f}(x, t)+a_{1} T_{m}(x, t)-u(t) \frac{\partial T_{f}}{\partial x}(x, t) & ] 0, L[\times] 0, T[ \\ \frac{\partial T_{m}}{\partial t}(x, t)=b_{1} T_{f}(x, t)+b_{2} T_{m}(x, t)+b_{3} & ] 0, L[\times] 0, T[ \\ T_{f}(0, t)=T_{f, 0} & ] 0, T[ \\ \left(T_{f}(x, 0), T_{m}(x, 0)\right)=\left(T_{f, 0}, T_{m, 0}\right) & ] 0, L[\end{cases}
$$

where $a, a_{1}, b_{1}, b_{2}$ and $b_{3}$ are empirically estimated coefficients, depending on the weather and the optical properties of the collectors, and assumed to be constant in our application due to the choice of a small time interval.

$u(t)\left(\mathrm{m} \mathrm{s}^{-1}\right)$ is the velocity of the heat-transfer fluid. In what follows $u(t)$ stands for the control.

In [8], El Jai and Chalqi provide simulations of the original model (1), where it is shown that, for a relatively small fluid velocity, the temperature of the receiver tube reaches rapidly a given value $T_{\max } \simeq 873 \mathrm{~K}\left(\simeq 600^{\circ} \mathrm{C}\right)$, due to the quick metal heat absorption. They deduce that the temperature of the receiver tube $T_{m}(.,$.$) can be as-$ sumed to be time-independent and close to $T_{\max }$, while the simulated fluid temperature remains very close to the one in the original model (1). Therefore, El Jai and Chalqi con- 
clude in [8] that the collector model (1) may be simplified into a system of one partial differential equation, given by

$$
\left\{\begin{array}{l}
\frac{\partial T_{f}}{\partial t}(x, t)=a T_{f}(x, t)+a_{1} T_{m}(x)-u(t) \frac{\partial T_{f}}{\partial x}(x, t) \\
T_{f}(0, t)=T_{f, 0} \\
T_{f}(x, 0)=T_{f, 0}
\end{array}\right.
$$

where the metallic tube temperature $T_{m}$ is approximated by a smooth function, satisfying $T_{m}(0)=-\frac{a}{a_{1}} T_{f, 0}$, as depicted in Fig. 2 .

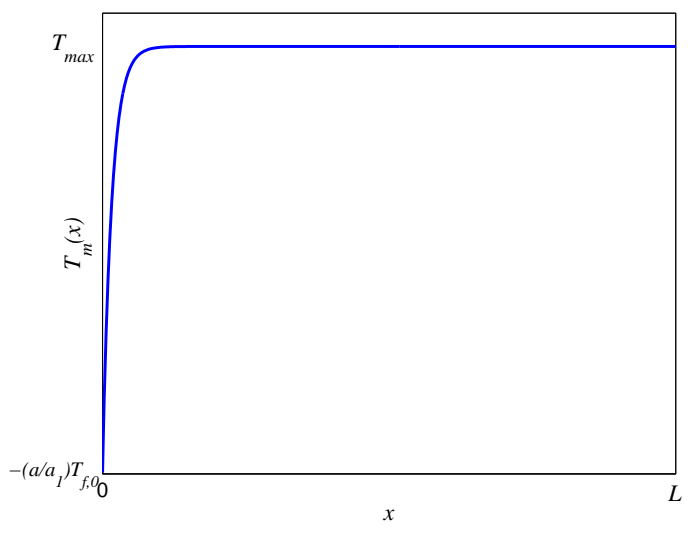

Figure 2. Temperature profile of the metallic tube $T_{m}$

We consider the state space $H=L^{2}(0, L)$, and we define the state $y(t) \in H$ by

$$
[y(t)](x)=T_{f}(x, t)-T_{f, 0}
$$

Denote $B=-\frac{\partial}{\partial x}$, and $b=a T_{f, 0}+a_{1} T_{m} \in H$, then equation (2) takes the form of the following bilinear system

$$
\left\{\begin{array}{l}
\dot{y}(t)=a y(t)+u(t) B y(t)+b \\
y(0)=0
\end{array}\right.
$$

The domain of $B$ is $\mathcal{D}(B)=\left\{z \in H^{1}(0, L): z(0)=0\right\}$, hence $y(0), b \in \mathcal{D}(B)$. Moreover, $B$ is the infinitesimal generator of the following $C_{0}$ semigroup

$$
[S(t) y](x)= \begin{cases}y(x-t) & \text { if } x-t>0 \\ 0 & \text { if } x-t<0\end{cases}
$$

For a maximal performance, the fluid temperature $T_{f}$ has to be close to a given value $T_{d}=673.15 \mathrm{~K}\left(400^{\circ} \mathrm{C}\right)$ along the tube. To this end, we will search the optimal fluid velocity $u($.$) that drives y(t)$ close to $y_{d}=T_{d}-T_{f, 0}$ by minimizing a quadratic cost functional. This is the purpose of the next section. 


\section{Optimal control problem}

We consider the following system

$$
\left\{\begin{array}{l}
\dot{y}(t)=a y(t)+u(t) B y(t)+b(t) \\
y(0)=y_{0}
\end{array}\right.
$$

where $B$ is the infinitesimal generator of a $C_{0}$ semigroup $(S(t))_{t \geq 0}$ on a separable Hilbert space $H$, with a dense domain $\mathcal{D}(B) . u \in L^{\infty}(0, T)$ is the control, such that $u(t) \geq 0$ almost everywhere (a.e.). $a \in \mathbb{R}, b \in L^{2}(0, T ; \mathcal{D}(B))$, and $y_{0} \in \mathcal{D}(B)$.

Our purpose is to minimize the following cost functional

$$
J(u)=\frac{\alpha}{2}\left\|y(T)-y_{d}\right\|^{2}+\frac{\beta}{2} \int_{0}^{T}\left\|y(s)-y_{d}\right\|^{2} d s+\frac{r}{2} \int_{0}^{T} u(s)^{2} d s
$$

over the set of admissible controls

$$
U_{a d}=\left\{u \in L^{\infty}(0, T): u_{\min } \leq u(t) \leq u_{\max } \text { a.e. }\right\}
$$

where $y_{d} \in H, \alpha, \beta \geq 0, r>0$, and $0 \leq u_{\min }<u_{\max }$.

If $r$ is small compared to $\frac{\max (\alpha, \beta)}{T u_{\max }^{2}}$, then the minimization of the cost functional (5) allows to drive the state $y(t)$ relatively close to $y_{d}$.

Thus, the optimal control problem is stated as :

$$
\left\{\begin{array}{l}
\min J(u) \\
u \in U_{a d}
\end{array}\right.
$$

We first give the expression of the unique mild solution of system (4).

Lemma 3.1. System (4) has a unique mild solution, written as

$$
y(t)=S\left(\int_{0}^{t} u(\tau) d \tau\right) y_{0}+\int_{0}^{t} S\left(\int_{s}^{t} u(\tau) d \tau\right)[a y(s)+b(s)] d s
$$

where $(S(t))_{t>0}$ is the $C_{0}$ semigroup generated by $B$.

Moreover, since $y_{0} \in \mathcal{D}(B)$, then $y(t) \in \mathcal{D}(B), \forall t \geq 0$.

Proof. For $u \in L^{\infty}(0, T)$ such that $u(t) \geq 0$ a.e., denote $U(t, s)=S\left(\int_{s}^{t} u(\tau) d \tau\right)$. The family of operators $(U(t, s))_{t \geq s}$ satisfies definition 9.2, chapter VI in [9], then $U(t, s)$ is a strongly continuous evolution family. Besides, there exist constants $M \geq 1$ and $\omega \in \mathbb{R}$ such that $\|U(t, s)\| \leq M e^{|\omega|\|u\|(t-s)}$, then $U(t, s)$ is exponentially bounded. Additionally, a simple calculus yields

$$
\frac{d}{d t} U(t, s) z=u(t) B U(t, s) z, \quad \forall t \geq s, \forall z \in \mathcal{D}(B)
$$

Then $(U(t, s))_{t \geq s}$ is generated by the family of operators $B(t)=u(t) B$.

By virtue of corollary 9.20, chapter VI in [9], it follows that the family of operators $(u(t) B+a I)_{t \geq 0}$ generates an exponentially bounded evolution family $(\Gamma(t, s))_{t \geq s \geq 0}$, given by

$$
\Gamma(t, s) z=U(t, s) z+\int_{s}^{t} U(t, \tau) a \Gamma(\tau, s) z d \tau, \quad \forall z \in H
$$


Then system (4) has a unique mild solution, written as

$$
y(t)=\Gamma(t, 0) y_{0}+\int_{0}^{t} \Gamma(t, s) b(s) d s
$$

Replacing $\Gamma(t, 0)$ and $\Gamma(t, s)$ by their respective expressions, and applying Fubini's theorem yield

$$
y(t)=U(t, 0) y_{0}+\int_{0}^{t} U(t, s)[a y(s)+b(s)] d s
$$

Hence we obtain (8). Finally, since $y_{0} \in \mathcal{D}(B)$ then, by proposition 9.3, chapter VI in [9], $y(t) \in \mathcal{D}(B), \forall t \geq 0$.

In the next proposition we prove the existence of an optimal control.

Proposition 3.2. There exists $u^{*} \in U_{a d}$ such that $J\left(u^{*}\right)=\inf _{u \in U_{a d}} J(u)$.

Proof. The functional $J$ is nonnegative, and the set $U_{a d}$ is nonempty, then there exists $J^{*} \geq 0$ such that $J^{*}=\inf \left\{J(u) \mid u \in U_{a d}\right\}$. Let $\left(u_{n}\right)_{n \in \mathbb{N}}$ be a sequence in $U_{a d}$, such that $\lim _{n \rightarrow \infty} J\left(u_{n}\right)=J^{*}$. By the boundedness of $U_{a d}$, the sequence $\left(u_{n}\right)_{n \in \mathbb{N}}$ is bounded, then $\left(u_{n}\right)_{n \in \mathbb{N}}$ has a subsequence, still denoted $\left(u_{n}\right)_{n \in \mathbb{N}}$, converging weakly to $u^{*}$ in $L^{2}(0, T)$.

According to the partial converse of Lebesgue theorem (see theorem 4.9 in [5]), any sequence $\left(v_{n}\right)_{n \in \mathbb{N}}$ in $U_{a d}$, such that $v_{n} \rightarrow \bar{v}$ in $L^{2}(0, T)$, has a subsequence $\left(v_{n_{k}}\right)_{n \in \mathbb{N}}$ satisfying $v_{n_{k}}(t) \rightarrow \bar{v}(t)$ a.e. on $[0, T]$. Since $u_{\min } \leq v_{n_{k}}(t) \leq u_{\max }$ a.e., then $u_{\min } \leq \bar{v}(t) \leq u_{\max }$ a.e. on $[0, T]$, which yields $\bar{v} \in U_{a d}$. It follows that $U_{a d}$ is closed in $L^{2}(0, T)$. Additionally, $U_{a d}$ is convex, hence $U_{a d}$ is weakly closed in $L^{2}(0, T)$. Therefore $u^{*} \in U_{a d}$.

Let $y_{n}$ and $y^{*}$ be the respective mild solutions of system (4), relatively to $u_{n}$ and $u^{*}$. Then

$$
\begin{aligned}
y_{n}(t)-y^{*}(t)= & S\left(\int_{0}^{t} u_{n}(\tau) d \tau\right) y_{0}-S\left(\int_{0}^{t} u^{*}(\tau) d \tau\right) y_{0} \\
& +\int_{0}^{t} S\left(\int_{s}^{t} u_{n}(\tau) d \tau\right) a\left[y_{n}(s)-y^{*}(s)\right] d s \\
& +\int_{0}^{t}\left[S\left(\int_{s}^{t} u_{n}(\tau) d \tau\right) y^{*}(s)-S\left(\int_{s}^{t} u^{*}(\tau) d \tau\right) y^{*}(s)\right] d s \\
& +\int_{0}^{t}\left[S\left(\int_{s}^{t} u_{n}(\tau) d \tau\right) b(s)-S\left(\int_{s}^{t} u^{*}(\tau) d \tau\right) b(s)\right] d s
\end{aligned}
$$

Denote $\mu=\sup _{n \in \mathbb{N}}\left\|u_{n}\right\|_{L^{\infty}(0, T)}$, and let $M \geq 1$ and $\omega \in \mathbb{R}$ such that $\|S(t)\| \leq M e^{\omega t}$, $\forall t \geq 0$. Let $\widehat{M \in \mathbb{N}}=M e^{|\omega a| T \mu}$. Then applying Gronwall's lemma to the above equality yields

$$
\begin{aligned}
\left\|y_{n}(t)-y^{*}(t)\right\| \leq & \widehat{M} \\
& +\widehat{M}\left(\int_{0}^{t} u_{n}(\tau) d \tau\right) y_{0}-S\left(\int_{0}^{t} u^{*}(\tau) d \tau\right) y_{0} \| \\
& \left.+\widehat{M}\left\|\int_{0}^{t}\left[S\left(\int_{s}^{t} u_{n}(\tau) d \tau\right) y_{n}^{*}(s)-S\left(\int_{s}^{t} u^{*}(\tau) d \tau\right) y^{*}(s)\right] d s\right\| b(s)-S\left(\int_{s}^{t} u^{*}(\tau) d \tau\right) b(s)\right] d s \|
\end{aligned}
$$


The weak convergence $u_{n} \rightarrow u^{*}$ gives $\lim _{n \rightarrow \infty} \int_{s}^{t} u_{n}(\tau) d \tau=\int_{s}^{t} u^{*}(\tau) d \tau, \quad \forall s, t \in[0, T]$. It follows that

$$
\begin{gathered}
\lim _{n \rightarrow \infty}\left\|S\left(\int_{0}^{t} u_{n}(\tau) d \tau\right) y_{0}-S\left(\int_{0}^{t} u^{*}(\tau) d \tau\right) y_{0}\right\|=0 \\
\lim _{n \rightarrow \infty}\left\|S\left(\int_{s}^{t} u_{n}(\tau) d \tau\right) y^{*}(s)-S\left(\int_{s}^{t} u^{*}(\tau) d \tau\right) y^{*}(s)\right\|=0 \quad \text { a.e. on }[0, t] \\
\lim _{n \rightarrow \infty}\left\|S\left(\int_{s}^{t} u_{n}(\tau) d \tau\right) b(s)-S\left(\int_{s}^{t} u^{*}(\tau) d \tau\right) b(s)\right\|=0 \quad \text { a.e. on }[0, t]
\end{gathered}
$$

Then, by applying Lebesgue's dominated convergence theorem, we obtain

$$
\begin{gathered}
\lim _{n \rightarrow \infty}\left\|\int_{0}^{t}\left[S\left(\int_{s}^{t} u_{n}(\tau) d \tau\right) y^{*}(s)-S\left(\int_{s}^{t} u^{*}(\tau) d \tau\right) y^{*}(s)\right] d s\right\|=0 \\
\lim _{n \rightarrow \infty}\left\|\int_{0}^{t}\left[S\left(\int_{s}^{t} u_{n}(\tau) d \tau\right) b(s)-S\left(\int_{s}^{t} u^{*}(\tau) d \tau\right) b(s)\right] d s\right\|=0
\end{gathered}
$$

Therefore $y_{n}(t) \rightarrow y^{*}(t)$ strongly in $H$, for every $t \in[0, T]$.

By Fatou's lemma, we obtain

$$
\int_{0}^{T}\left\|y^{*}(t)-y_{d}\right\|^{2} d t \leq \liminf _{n \rightarrow \infty} \int_{0}^{T}\left\|y_{n}(t)-y_{d}\right\|^{2} d t
$$

and by the lower semi-continuity of norms, we have

$$
\int_{0}^{T} u^{*}(t)^{2} d t \leq \liminf _{n \rightarrow \infty} \int_{0}^{T} u_{n}(t)^{2} d t
$$

Hence $J\left(u^{*}\right) \leq \liminf _{n \rightarrow \infty} J\left(u_{n}\right)=J^{*}$, which yields $J\left(u^{*}\right)=J^{*}$.

Hereafter we prove some technical results, that will be useful to formulate an optimality condition.

Proposition 3.3. Let $u, h \in L^{\infty}(0, T)$ such that $u(t), h(t) \geq 0$ a.e., then

$$
\lim _{\varepsilon \rightarrow 0^{+}} \frac{y_{u+\varepsilon h}(t)-y_{u}(t)}{\varepsilon}=z_{h}(t)
$$

where $z_{h}$ is the mild solution of the following equation :

$$
\left\{\begin{array}{l}
\dot{z}_{h}(t)=a z_{h}(t)+u(t) B z_{h}(t)+h(t) B y_{u}(t) \\
z_{h}(0)=0
\end{array}\right.
$$

and is written as :

$$
z_{h}(t)=\int_{0}^{t} S\left(\int_{s}^{t} u(\tau) d \tau\right)\left[a z_{h}(s)+h(s) B y_{u}(s)\right] d s
$$


Proof. Let $u, h \in L^{\infty}(0, T)$, such that $u(t), h(t) \geq 0$ a.e. on $[0, T]$. Let $\varepsilon>0$. Without loss of generality, we can assume that $\varepsilon\|h\| \leq 1$.

Let $y_{u}$ and $y_{u+\varepsilon h}$ be the respective mild solutions of system (4), relatively to $u$ and $u+\varepsilon h$, and let $z_{h}$ be the mild solution of equation (10). Denote $U(t, s)=S\left(\int_{s}^{t} u(\tau) d \tau\right)$. By replacing $y_{u}(s)$ in equation (11) by its expression, we obtain

$$
\begin{aligned}
z_{h}(t)= & \int_{0}^{t} U(t, s) a z_{h}(s) d s+\int_{0}^{t} h(s) B U(t, 0) y_{0} d s \\
& +\int_{0}^{t} \int_{0}^{s} h(s) B U(t, \theta)\left[a y_{u}(\theta)+b(\theta)\right] d \theta d s
\end{aligned}
$$

Applying Fubini's theorem to the above expression yields

$$
\begin{aligned}
z_{h}(t) & =\int_{0}^{t} U(t, s) a z_{h}(s) d s+\left(\int_{0}^{t} h(\tau) d \tau\right) B U(t, 0) y_{0} \\
& +\int_{0}^{t}\left(\int_{s}^{t} h(\theta) d \theta\right) B U(t, s)\left[a y_{u}(s)+b(s)\right] d s
\end{aligned}
$$

For $0 \leq s \leq t \leq T$, we denote

$$
\left\{\begin{array}{l}
Y_{0}(t)=U(t, 0) y_{0} \\
Y(s)=U(t, s) a y_{u}(s) \\
Z(s)=U(t, s) b(s) \\
H_{\varepsilon}(t, s)=S\left(\varepsilon \int_{s}^{t} h(\tau) d \tau\right)
\end{array}\right.
$$

Then

$$
\begin{aligned}
& y_{u}(t)=Y_{0}(t)+\int_{0}^{t}[Y(s)+Z(s)] d s \\
& y_{u+\varepsilon h}(t)=H_{\varepsilon}(t, 0) Y_{0}(t)+\int_{0}^{t} H_{\varepsilon}(t, s) U(t, s) a y_{u+\varepsilon h}(s) d s+\int_{0}^{t} H_{\varepsilon}(t, s) Z(s) d s
\end{aligned}
$$

and, by expression (12), we have

$$
z_{h}(t)=\int_{0}^{t} U(t, s) a z_{h}(s) d s+\left(\int_{0}^{t} h(\tau) d \tau\right) B Y_{0}(t)+\int_{0}^{t}\left(\int_{s}^{t} h(\tau) d \tau\right) B[Y(s)+Z(s)] d s
$$

which yields

$$
\begin{aligned}
\frac{y_{u+\varepsilon h}(t)-y_{u}(t)}{\varepsilon}-z_{h}(t) & =\frac{H_{\varepsilon}(t, 0) Y_{0}(t)-Y_{0}(t)}{\varepsilon}-\int_{0}^{t} h(\tau) d \tau B Y_{0}(t) \\
& +\int_{0}^{t}\left[\frac{H_{\varepsilon}(t, s) Y(s)-Y(s)}{\varepsilon}-\int_{s}^{t} h(\tau) d \tau B Y(s)\right] d s \\
& +\int_{0}^{t}\left[\frac{H_{\varepsilon}(t, s) Z(s)-Z(s)}{\varepsilon}-\int_{s}^{t} h(\tau) d \tau B Z(s)\right] d s \\
& +\int_{0}^{t} H_{\varepsilon}(t, s) U(t, s) a\left[\frac{y_{u+\varepsilon h}(s)-y_{u}(s)}{\varepsilon}-z_{h}(s)\right] d s \\
& +\int_{0}^{t}\left[H_{\varepsilon}(t, s) a z_{h}(s)-a z_{h}(s)\right] d s
\end{aligned}
$$


There exist $M \geq 1$ and $\omega \in \mathbb{R}$ such that $\|S(t)\| \leq M e^{\omega t}, \forall t \geq 0$. Then applying Gronwall's lemma to the above equality yields

$$
\begin{aligned}
\left\|\frac{y_{u+\varepsilon h}(t)-y_{u}(t)}{\varepsilon}-z_{h}(t)\right\| & \leq \widehat{M}\left\|\frac{H_{\varepsilon}(t, 0) Y_{0}(t)-Y_{0}(t)}{\varepsilon}-\int_{0}^{t} h(\tau) d \tau B Y_{0}(t)\right\| \\
& +\widehat{M}\left\|\int_{0}^{t}\left[\frac{H_{\varepsilon}(t, s) Y(s)-Y(s)}{\varepsilon}-\int_{s}^{t} h(\tau) d \tau B Y(s)\right] d s\right\| \\
& +\widehat{M}\left\|\int_{0}^{t}\left[\frac{H_{\varepsilon}(t, s) Z(s)-Z(s)}{\varepsilon}-\int_{s}^{t} h(\tau) d \tau B Z(s)\right] d s\right\| \\
& +\widehat{M}\left\|\int_{0}^{t}\left[H_{\varepsilon}(t, s) a z_{h}(s)-a z_{h}(s)\right] d s\right\|
\end{aligned}
$$

where $\widehat{M}=M e^{|\omega a| T(\|u\|+1)}$. By passing to the limit, we have

$$
\lim _{\varepsilon \rightarrow 0}\left\|H_{\varepsilon}(t, s) a z_{h}(s)-a z_{h}(s)\right\|=0 \quad \text { a.e. on }[0, t]
$$

Since $Y_{0}(t), Y(s)$ and $Z(s)$ belong to $\mathcal{D}(B)$ then, by using the semigroup property $\lim _{\tau \rightarrow 0^{+}} \frac{S(\tau) z-z}{\tau}=B z, \forall z \in \mathcal{D}(B)$, we obtain

$$
\begin{gathered}
\lim _{\varepsilon \rightarrow 0^{+}}\left\|\frac{H_{\varepsilon}(t, 0) Y_{0}(t)-Y_{0}(t)}{\varepsilon}-\int_{0}^{t} h(\tau) d \tau B Y_{0}(t)\right\|=0 \\
\lim _{\varepsilon \rightarrow 0^{+}}\left\|\frac{H_{\varepsilon}(t, s) Y(s)-Y(s)}{\varepsilon}-\int_{s}^{t} h(\tau) d \tau B Y(s)\right\|=0 \quad \text { a.e. on }[0, t] \\
\lim _{\varepsilon \rightarrow 0^{+}}\left\|\frac{H_{\varepsilon}(t, s) Z(s)-Z(s)}{\varepsilon}-\int_{s}^{t} h(\tau) d \tau B Z(s)\right\|=0 \quad \text { a.e. on }[0, t]
\end{gathered}
$$

By using appropriate bounds, and applying the dominated convergence theorem, we have

$$
\begin{gathered}
\lim _{\varepsilon \rightarrow 0^{+}}\left\|\int_{0}^{t}\left[H_{\varepsilon}(t, s) a z_{h}(s)-a z_{h}(s)\right] d s\right\|=0 \\
\lim _{\varepsilon \rightarrow 0^{+}}\left\|\int_{0}^{t}\left[\frac{H_{\varepsilon}(t, s) Y(s)-Y(s)}{\varepsilon}-\int_{s}^{t} h(\tau) d \tau B Y(s)\right] d s\right\|=0 \\
\lim _{\varepsilon \rightarrow 0^{+}}\left\|\int_{0}^{t}\left[\frac{H_{\varepsilon}(t, s) Z(s)-Z(s)}{\varepsilon}-\int_{s}^{t} h(\tau) d \tau B Z(s)\right] d s\right\|=0
\end{gathered}
$$

Therefore

$$
\lim _{\varepsilon \rightarrow 0^{+}}\left\|\frac{y_{u+\varepsilon h}(t)-y_{u}(t)}{\varepsilon}-z_{h}(t)\right\|=0
$$

Corollary 3.4. Let $u \in L^{\infty}(0, T)$ such that $u(t) \geq 0$ a.e.

For any $h \in L^{\infty}(0, T)$ satisfying $h(t) \leq 0$ and $h(t)+u(t) \geq 0$ a.e., we have

$$
\lim _{\varepsilon \rightarrow 0^{+}} \frac{y_{u+\varepsilon h}(t)-y_{u}(t)}{\varepsilon}=z_{h}(t)
$$

where $z_{h}$ is the mild solution of (10), and satisfies expression (11). 
Proof. Let $h \in L^{\infty}(0, T)$ such that $h(t) \leq 0$ and $u(t)+h(t) \geq 0$ a.e. Let $0<\varepsilon \leq 1$, and denote $v=u+\varepsilon h$. Then

$$
\frac{y_{u+\varepsilon h}(t)-y_{u}(t)}{\varepsilon}=-\frac{y_{v+\varepsilon(-h)}(t)-y_{v}(t)}{\varepsilon}
$$

By proposition 3.2, we have

$$
\frac{y_{v+\varepsilon(-h)}(t)-y_{v}(t)}{\varepsilon}=z_{(-h)}^{\varepsilon}(t)+\theta(\varepsilon)
$$

where $\lim _{\varepsilon \rightarrow 0} \theta(\varepsilon)=0$, and $z_{(-h)}^{\varepsilon}$ is the solution of equation (10), with $v$ and $-h$ instead of $u$ and $h$, respectively. A standard calculus leads to

$$
\lim _{\varepsilon \rightarrow 0^{+}} z_{(-h)}^{\varepsilon}(t)=-z_{h}(t)
$$

Hence

$$
\lim _{\varepsilon \rightarrow 0^{+}} \frac{y_{v+\varepsilon(-h)}(t)-y_{v}(t)}{\varepsilon}=-z_{h}(t)
$$

which yields the limit (13).

Proposition 3.5. Let $u \in L^{\infty}(0, T)$ such that $u(t) \geq 0$ a.e.

For any $h \in L^{\infty}(0, T)$ of constant sign, satisfying $u(t)+h(t) \geq 0$ a.e., we have

$$
\lim _{\varepsilon \rightarrow 0^{+}} \frac{J(u+\varepsilon h)-J(u)}{\varepsilon}=\int_{0}^{T} J_{u}^{\prime}(t) h(t) d t
$$

where

$$
J_{u}^{\prime}(t)=\langle\varphi(t), B y(t)\rangle_{H}+r u(t)
$$

such that $y$ is the mild solution of system (4), associated with $u$, and $\varphi$ is the mild solution of the following adjoint equation

$$
\left\{\begin{array}{l}
\dot{\varphi}(t)=-a \varphi(t)-u(t) B^{*} \varphi(t)-\beta\left(y(t)-y_{d}\right) \\
\varphi(T)=\alpha\left(y(T)-y_{d}\right)
\end{array}\right.
$$

$B^{*}$ denotes the adjoint operator of $B$.

Proof. Let $h \in L^{\infty}(0, T)$ have a constant sign, and such that $u(t)+h(t) \geq 0$ a.e. Using the limits (9) and (13), we have

$$
\begin{aligned}
\lim _{\varepsilon \rightarrow 0^{+}} \frac{J(u+\varepsilon h)-J(u)}{\varepsilon}= & \left\langle\alpha\left(y_{u}(T)-y_{d}\right), z_{h}(T)\right\rangle_{H}+\int_{0}^{T}\left\langle\beta\left(y_{u}(t)-y_{d}\right), z_{h}(t)\right\rangle_{H} d t \\
& +\int_{0}^{T} r u(t) h(t) d t
\end{aligned}
$$

For $n \in \rho(B)$, the resolvent set of $B$, let $B_{n}=n B(n I-B)^{-1}$ be the Yosida approximation of $B$. Let $z_{n}$ and $\varphi_{n}$ be the respective mild solutions of the following equations

$$
\begin{gathered}
\left\{\begin{array}{l}
\dot{z}_{n}(t)=a z_{n}(t)+u(t) B_{n} z_{n}(t)+h(t) B y_{u}(t) \\
z_{h}(0)=0
\end{array}\right. \\
\left\{\begin{array}{l}
\dot{\varphi}_{n}(t)=-a \varphi_{n}(t)-u(t) B_{n}^{*} \varphi_{n}(t)-\beta\left(y_{u}(t)-y_{d}\right) \\
\varphi_{n}(T)=\alpha\left(y_{u}(T)-y_{d}\right)
\end{array}\right.
\end{gathered}
$$


$B_{n}^{*}$ is bounded, then $\mathcal{D}\left(B_{n}^{*}\right)=H$, leading to $B_{n}^{*} \varphi_{n}(t) \in H$ a.e. on $[0, T]$. In addition, the definition in p. 370 of [3] yields

$$
\left\{\begin{array}{l}
\frac{d}{d t}\left\langle z_{n}(t), \psi\right\rangle=\left\langle a z_{n}(t)+u(t) B_{n} z_{n}(t)+h(t) B y_{u}(t), \psi\right\rangle \\
\frac{d}{d t}\left\langle\varphi_{n}(t), \psi\right\rangle=\left\langle-a \varphi_{n}(t)-u(t) B_{n}^{*} \varphi_{n}(t)-\beta\left(y_{u}(t)-y_{d}\right), \psi\right\rangle
\end{array} \quad, \quad \text { a.e. on }[0, T]\right.
$$

for every $\psi \in H$. It follows that

$$
\left\{\begin{array}{l}
\left\langle\dot{z}_{n}(.), \psi\right\rangle=\frac{d}{d t}\left\langle z_{n}(.), \psi\right\rangle \in L^{2}(0, T) \\
\left\langle\dot{\varphi}_{n}(.), \psi\right\rangle=\frac{d}{d t}\left\langle\varphi_{n}(.), \psi\right\rangle \in L^{2}(0, T)
\end{array}, \quad \forall \psi \in H\right.
$$

Therefore, $\dot{z}_{n}, \dot{\varphi}_{n} \in L^{2}(0, T, H)$, which leads to the following calculus

$$
\begin{aligned}
& \int_{0}^{T}\left\langle\beta\left(y_{u}(t)-y_{d}\right), z_{n}(t)\right\rangle_{H} d t=-\int_{0}^{T}\left\langle\dot{\varphi}_{n}(t)+u(t) B_{n}^{*} \varphi_{n}(t)+a \varphi_{n}(t), z_{n}(t)\right\rangle_{H} d t \\
& =-\int_{0}^{T}\left\langle\dot{\varphi}_{n}(t), z_{n}(t)\right\rangle_{H} d t-\int_{0}^{T}\left\langle\varphi_{n}(t), u(t) B_{n} z_{n}(t)+a z_{n}(t)\right\rangle_{H} d t \\
& =-\int_{0}^{T}\left\langle\dot{\varphi}_{n}(t), z_{n}(t)\right\rangle_{H} d t-\int_{0}^{T}\left\langle\varphi_{n}(t), \dot{z}_{n}(t)\right\rangle_{H} d t+\int_{0}^{T}\left\langle\varphi_{n}(t), h(t) B y_{u}(t)\right\rangle_{H} d t
\end{aligned}
$$

Since $\dot{z}_{n}, \dot{\varphi}_{n} \in L^{2}(0, T ; H)$, then

$$
\begin{aligned}
\int_{0}^{T}\left\langle\dot{\varphi}_{n}(t), z_{n}(t)\right\rangle_{H} d t+\int_{0}^{T}\left\langle\varphi_{n}(t), \dot{z}_{n}(t)\right\rangle_{H} d t & =\left\langle\varphi_{n}(T), z_{n}(T)\right\rangle_{H}-\left\langle\varphi_{n}(0), z_{n}(0)\right\rangle_{H} \\
& =\left\langle\alpha\left(y_{u}(T)-y_{d}\right), z_{n}(T)\right\rangle_{H}
\end{aligned}
$$

It follows that

$$
\int_{0}^{T}\left\langle\beta\left(y_{u}(t)-y_{d}\right), z_{n}(t)\right\rangle_{H} d t=-\left\langle\alpha\left(y_{u}(T)-y_{d}\right), z_{n}(T)\right\rangle_{H}+\int_{0}^{T}\left\langle\varphi_{n}(t), h(t) B y_{u}(t)\right\rangle_{H} d t
$$

By virtue of proposition 5.4, chapter 2 of [12], we have

$$
\lim _{n \rightarrow+\infty}\left\|z_{n}-z_{h}\right\|_{C([0, T] ; H)}=0 \text { and } \lim _{n \rightarrow+\infty}\left\|\varphi_{n}-\varphi\right\|_{C([0, T] ; H)}=0
$$

where $z_{h}$ and $\varphi$ are the respective mild solutions of (10) and (15). Then the above equality yields

$$
\int_{0}^{T}\left\langle\beta\left(y_{u}(t)-y_{d}\right), z_{h}(t)\right\rangle_{H} d t+\left\langle\alpha\left(y_{u}(T)-y_{d}\right), z_{h}(T)\right\rangle_{H}=\int_{0}^{T}\left\langle\varphi(t), B y_{u}(t)\right\rangle_{H} h(t) d t
$$

Therefore

$$
\lim _{\varepsilon \rightarrow 0^{+}} \frac{J(u+\varepsilon h)-J(u)}{\varepsilon}=\int_{0}^{T}\left[\left\langle\varphi(t), B y_{u}(t)\right\rangle_{H}+r u(t)\right] h(t) d t
$$


In the next proposition we formulate a necessary optimality condition.

Proposition 3.6. Let $u^{*}$ be an optimal control, then $u^{*}$ satisfies :

$$
u^{*}(t)=\max \left(u_{\min } ; \min \left(u_{\max } ;-\frac{1}{r}\langle\varphi(t), B y(t)\rangle_{H}\right)\right)
$$

where $y$ and $\varphi$ are respectively the mild solutions of (4) and (15), associated with $u^{*}$.

Proof. Let $u^{*}$ be an optimal control, and let $h \in L^{\infty}(0, T)$ have a constant sign, and such that $u^{*}+h \in U_{a d}$, where $U_{a d}$ is given by (6). The convexity of $U_{a d}$ yields $u^{*}+\varepsilon h \in U_{a d}$, $\forall \varepsilon \in] 0,1]$. Hence

$$
\left.\left.J\left(u^{*}+\varepsilon h\right) \geq J\left(u^{*}\right), \quad \forall \varepsilon \in\right] 0,1\right]
$$

Using proposition 3.5 , we obtain

$$
\int_{0}^{T} J_{u^{*}}^{\prime}(t) h(t) d t=\lim _{\varepsilon \rightarrow 0^{+}} \frac{J\left(u^{*}+\varepsilon h\right)-J\left(u^{*}\right)}{\varepsilon} \geq 0
$$

- If $u_{\min }<u^{*}(t)<u_{\max }$ over a nonempty open set $\left.I \subset\right] 0, T[$ :

Let $h \in \mathscr{D}(I)$ (the space of smooth functions, compactly supported) of constant sign. If $\|h\|_{L^{\infty}(I)}$ is sufficiently small, then $u^{*}+h, u^{*}-h \in U_{a d}$. It follows from inequality (17), applied to $h$ and $-h$, that

$$
\int_{I} J_{u^{*}}^{\prime}(t) h(t) d t=0, \quad \forall h \in \mathscr{D}(I) \text { of constant sign, }
$$

which yields $J_{u^{*}}^{\prime}(t)=0$ a.e. on $I$. Then by expression (14) we have

$$
u^{*}(t)=-\frac{1}{r}\langle\varphi(t), B y(t)\rangle \text { a.e. on } I
$$

- If $u^{*}(t)=u_{\text {min }}$ over a nonempty open set $\left.I \subset\right] 0, T[$ :

Let $h \in \mathscr{D}(I)$ such that $h(t) \geq 0, \forall t \in I$. Then for $\|h\|_{L^{\infty}(I)}$ sufficiently small we have $u^{*}+h \in U_{a d}$. Hence inequality (17) yields $\int_{I} J_{u^{*}}^{\prime}(t) h(t) d t \geq 0$.

It follows that $J_{u^{*}}^{\prime}(t) \geq 0$ a.e. on $I$. Thus

$$
-\frac{1}{r}\langle\varphi(t), B y(t)\rangle_{H} \leq u_{\min }=u^{*}(t) \text { a.e. on } I
$$
then

- Similarly to the above case, if $u^{*}(t)=u_{\max }$ over a nonempty open set $\left.I \subset\right] 0, T[$,

$$
-\frac{1}{r}\langle\varphi(t), B y(t)\rangle_{H} \geq u_{\max } \text { a.e. on } I
$$

Therefore, $u^{*}(t)=\max \left(u_{\min } ; \min \left(u_{\max } ;-\frac{1}{r}\langle\varphi(t), B y(t)\rangle_{H}\right)\right)$ a.e. on $[0, T]$.

Remarks 3.7. 1) If $u^{*}$ is an optimal control, then a simple calculus leads to

$$
\begin{aligned}
& \alpha\left\|y^{*}(T)-y_{d}\right\|^{2}+\beta \int_{0}^{T}\left\|y^{*}(s)-y_{d}\right\|^{2} d s \leq \\
& \leq \inf _{u \in U_{a d}}\left[\alpha\left\|y_{u}(T)-y_{d}\right\|^{2}+\beta \int_{0}^{T}\left\|y_{u}(s)-y_{d}\right\|^{2} d s\right]+\frac{r T u_{\max }^{2}}{\max (\alpha, \beta)}
\end{aligned}
$$


Particularly, if $\beta=0$, then the above inequality becomes

$$
\inf _{u \in U_{a d}}\left\|y_{u}(T)-y_{d}\right\|^{2} \leq\left\|y^{*}(T)-y_{d}\right\|^{2} \leq \inf _{u \in U_{a d}}\left\|y_{u}(T)-y_{d}\right\|^{2}+\frac{r T u_{\max }^{2}}{\alpha}
$$

Thus, choosing $r$ small with respect to $\frac{\max (\alpha, \beta)}{T u_{\max }^{2}}$ makes $\left\|y^{*}(T)-y_{d}\right\|$ close to the minimal value of $\left\|y_{u}(T)-y_{d}\right\|$ on $U_{a d}$.

2) Going back to the collector model (2), Proposition 3.6 shows that the optimal fluid velocity is written as

$$
u^{*}(t)=\max \left(u_{\min } ; \min \left(u_{\max } ; \int_{0}^{L} \psi(x, t) \frac{\partial T_{f}}{\partial x}(x, t) d x\right)\right) \quad\left(\mathrm{m} \mathrm{s}^{-1}\right)
$$

where $\frac{\partial T_{f}}{\partial x}\left({ }^{\circ} \mathrm{C} \mathrm{m}^{-1}\right)$ is the gradient of the fluid temperature, and $\psi\left(\mathrm{m} \mathrm{s}^{-1}{ }^{\circ} \mathrm{C}^{-1}\right)$ is the solution of the following equation

$$
\left\{\begin{array}{l}
\frac{\partial \psi}{\partial t}(x, t)=-a \psi(x, t)-u^{*}(t) \frac{\partial \psi}{\partial x}(x, t)-\frac{\beta}{r}\left(T_{f}(x, t)-T_{d}\right) \\
\psi(L, t)=0 \\
\psi(x, T)=\frac{\alpha}{r}\left(T_{f}(x, T)-T_{d}\right)
\end{array}\right.
$$

Note that $\psi=\frac{1}{r} \varphi$, where $\varphi$ is the solution of the adjoint equation (15). On a practical note, $\psi$ models the velocity change with temperature, and describes the effect of the temperature gap $\left(T_{f}-T_{d}\right)$ on $u^{*}$. More precisely, $\psi(x, t)$ models the rate of change in the optimal velocity with respect to $\left(T_{f}(x, t)-T_{d}\right)$. Accordingly, the expression of the optimal velocity $u^{*}(t)$ involves the integral over $[0, L]$ of $\psi(x, t) \frac{\partial T_{f}}{\partial x}(x, t)$.

The optimality condition (16) shows that the optimal control $u^{*}$ is function of $y$ and $\varphi$, which themselves are function of $u^{*}$. Then the control cannot be directly computed by (16). For this reason, we introduce the following algorithm, to implement numerically the optimal control.

\section{Algorithm}

- Step 1 : Choose an initial control $u_{0} \in U_{a d}$, a threshold accuracy $\varepsilon>0$, and a step length $\lambda$. Initialize with $k=0$.

- Step 2 : Compute $y_{k}$, solution of (4), and $\varphi_{k}$, solution of (15), relatively to $u_{k}$.

- Step 3 : Compute

$$
\begin{gathered}
J_{u_{k}}^{\prime}(t)=\left\langle\varphi_{k}(t), B y_{k}(t)\right\rangle+r u_{k}(t) \\
u_{k+1}(t)=\max \left(u_{\min } ; \min \left(u_{\max } ; u_{k}(t)-\lambda J_{u_{k}}^{\prime}(t)\right)\right)
\end{gathered}
$$

- Step 4 : If $\left\|u_{k+1}-u_{k}\right\|>\varepsilon, k=k+1$, go to step 2. Otherwise $u^{*}=u_{k}$. 


\section{Simulations}

We consider the collector model (2) with data of Ain Beni Mathar solar plant, in Northeast Morocco (see [6]) :

$$
L=618 m, a=-0.030, a_{1}=0.024, T_{f, 0}=423.15 \mathrm{~K}\left(150^{\circ} \mathrm{C}\right)
$$

To determine the optimal fluid velocity, we consider system (3), and the optimal control problem (7) with the cost functional (5), where

$$
T=3600 \mathrm{~s}, \quad \alpha=3, \quad \beta=0.1, \quad r=5 \times 10^{4}, \quad y_{d}=T_{d}-T_{f, 0}=250
$$

We assume that $0 \leq u(t) \leq 10^{-2}$. Then the set of admissible controls is

$$
U_{a d}=\left\{u \in L^{\infty}(0, T) \mid 0 \leq u(t) \leq 10^{-2} \text { a.e. }\right\}
$$

Simulations are carried out using the previous algorithm, with $u_{0}=0, \lambda=10^{-6}$, and $\varepsilon=10^{-5}$.

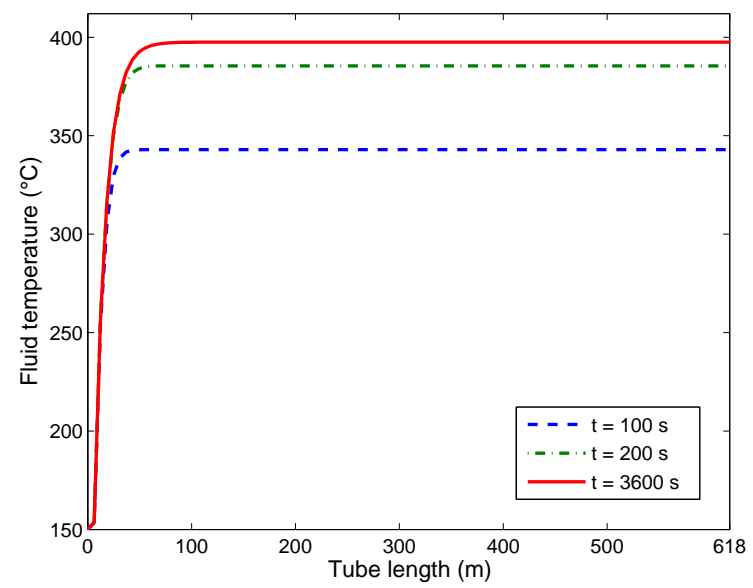

Figure 3. Fluid temperature at times $t=100 \mathrm{~s}, t=200 \mathrm{~s}$, and $t=3600 \mathrm{~s}$

Fig. 3 depicts the fluid temperature $\left({ }^{\circ} \mathrm{C}\right)$ at times $t=100 \mathrm{~s}, t=200 \mathrm{~s}$, and $t=T=3600 \mathrm{~s}$, while the corresponding optimal fluid velocity is plotted in Fig. 4. Fig. 3 shows that the fluid temperature gets close to the desired level $T_{d}=400^{\circ} \mathrm{C}$ within a short time interval. At the final time $T=3600$ s, the average fluid temperature is about $\widetilde{T} \simeq 390^{\circ} \mathrm{C}$.

As shown by simulations, the optimal fluid velocity, obtained with our approach, drives the fluid temperature close to the desired level. This is crucial for the performance of the solar plant.

\section{References}

[1] A. Addou, A. BEnBRik, "Existence and uniqueness of optimal control for a distributed parameter bilinear system”, J. Dyn. Control Syst., vol. 8, num. 2, pp. 141-152, 2002. 


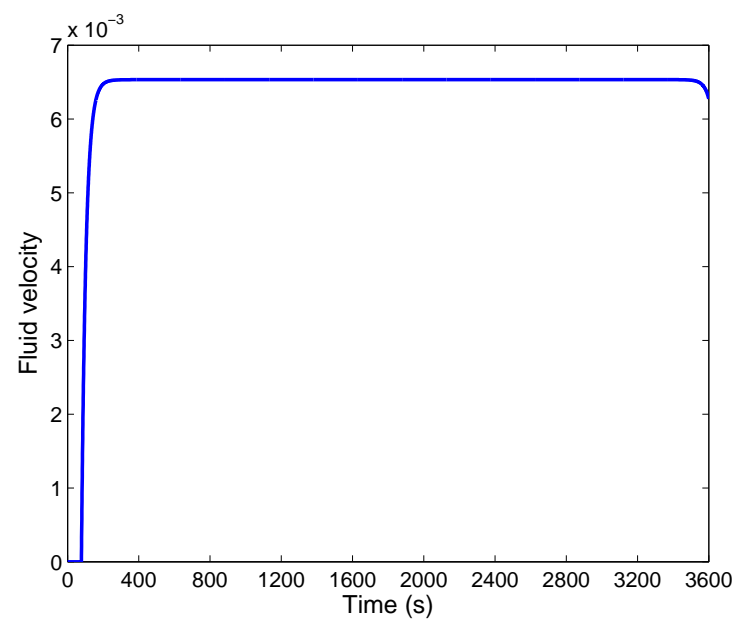

Figure 4. Optimal fluid velocity

[2] Alternative Energy Tutorials, http://www.alternative-energy-tutorials.com, accessed February 2018.

[3] J.M. BALL, "Strongly Continuous Semigroups, Weak Solutions, and the Variation of Constants Formula" Proc. Am. Math. Soc., vol. 63, num. 2, pp. 370-373, 1977.

[4] M.E. Bradley, S. Lenhart, J. Yong, "Bilinear optimal control of the velocity term in a Kirchhoff plate equation” J. Math. Anal. Appl., vol. 238, num. 2, pp. 451-467, 1999.

[5] H. BReZIS, "Functional Analysis, Sobolev Spaces and Partial Differential Equations", Springer-Verlag New York, 2010.

[6] F.Z. Chalqi, "Diverses approches de modélisation d'un capteur solaire cylindroparabolique", PhD thesis, University of Perpignan Via Domitia , 2013.

[7] J.-M. ClÉRIN, "Problèmes de contrôle optimal du type bilinéaire gouvernés par des équations aux dérivées partielles d'évolution. PhD thesis, University of Avignon, 2009. Available in http://tel.archives-ouvertes.fr

[8] M.C. El JAI, F.Z. CHALQI, "A modified model for parabolic trough solar receiver", AJER, vol. 2, pp. 200-211, 2013.

[9] J.C. Engel, R. NAGEL, “One-Parameter Semigroups for Linear Evolution Equations”, Graduate Texts in Mathematics, Springer-Verlag New York, 2000.

[10] R. ForRISTALL, "Heat transfer analysis and modeling of a parabolic trough solar receiver implemented in engineering equation solver", Technical report. National Renewable Energy laboratory, 2003.

[11] K. ITO, K. KUNISCH, "Optimal bilinear control of an abstract Schrödinger equation", SIAM J. Control Optim., vol. 46, num. 1, pp. 274-287, 2007.

[12] X. LI, J. Yong, "Optimal Control Theory for Infinite Dimensional Systems", Birkhäuser Boston, 1995.

[13] M. Liang, "Bilinear optimal control for a wave equation", Math. Mod. Meth. Appl. Sci., vol. 9, num. 1, pp. 45-68, 1999.

[14] E.H. Zerrik, N. El Boukhari, "Constrained optimal control for a class of semilinear infinite dimensional systems", J. Dyn. Control Syst., vol. 24, num. 1, pp. 65-81, 2018. 